TITLE:

\title{
Rovibrational transitions and nuclear spin conversion of methane in parahydrogen crystals
}

$\operatorname{AUTHOR}(S)$ :

Miki, M; Momose, T

CITATION:

Miki, M ... [et al]. Rovibrational transitions and nuclear spin conversion of methane in parahydrogen crystals. LOW TEMPERATURE PHYSICS 2000, 26(9-10): 661-668

\section{ISSUE DATE:}

2000

URL:

http://hdl.handle.net/2433/50038

\section{RIGHT:}

Copyright 2000 American Institute of Physics. This article may be downloaded for personal use only. Any other use requires prior permission of the author and the American Institute of Physics. 


\title{
Rovibrational transitions and nuclear spin conversion of methane in parahydrogen crystals
}

\author{
M. Miki and T. Momose*
} Division of Chemistry, Graduate School of Science, Kyoto University and Japan Science and Technology
Corporation (JST), Kyoto 606-8502, Japan

(Submitted February 7, 2000; revised May 25, 2000)

Fiz. Nizk. Temp. 26, 899-908 (September-October 2000)

Solid parahydrogen is an excellent matrix for matrix-isolation spectroscopy because of its high spectral resolution. Here we describe the rovibrational structure and nuclear spin conversion of $\mathrm{CH}_{4}$ embedded in parahydrogen crystals studied by infrared absorption spectroscopy. The vibration-rotation absorptions of $\mathrm{CH}_{4}$ exhibit time-dependent intensity changes at 4.8 $\mathrm{K}$. These changes are interpreted to be a result of the $I=1 \rightarrow I=2$ nuclear spin conversion that accompanies the $J=1 \rightarrow J=0$ rotational relaxation. The half-lifetime of the upper $J=1$ rotational state is unchanged by the addition of up to $2 \%$ orthohydrogen molecules but decreases with more than $10 \%$ orthohydrogen molecules. The increase of the decay rate at higher orthohydrogen concentration indicates that the magnetic field gradient across $\mathrm{CH}_{4}$ due to the orthohydrogen molecules mixes the nuclear spin states, which accelerates the conversion. (C) 2000 American Institute of Physics. [S1063-777X(00)00609-5]

\section{INTRODUCTION}

Matrix isolation spectroscopy at cryogenic temperatures has grown to be a methodology for a variety of applications in the field of molecular spectroscopy. Its application to the study of unstable molecules has piloted gas-phase spectroscopy. ${ }^{1}$ Not only unstable but also stable molecules in cryogenic matrices have been the subject of studies for understanding physics and chemistry in the condensed phase. ${ }^{2}$

In the early works by Lewis in the 1940s, organic molecular solids were used as the isolation matrices. ${ }^{3}$ Rare gas matrices, which were introduced by Pimentel and his co-workers, ${ }^{4}$ have been widely used in recent studies because of their chemically inert property and weak perturbations. The interaction from the environment, however, is not small due to the proximity of surrounding atoms and molecules, which makes the spectral linewidths of matrix-isolated species broader than those in the gas phase. The typical linewidth of vibrational transitions in rare gas matrices is on the order of $0.1-1 \mathrm{~cm}^{-1}$. The spectra in the condensed phase must contain much important information, such as intermolecular interactions and hindered motion of molecules under perturbation of the surrounding electrostatic potentials. Unfortunately, the broadening of the spectra wipes out most of the fine spectral structures containing such information.

Recently, it was found that the spectra of molecules in parahydrogen crystals are surprisingly sharp, as was initially noted by Oka and his co-workers. ${ }^{5-7}$ They studied parahydrogen crystals using high-resolution infrared and Raman spectroscopy and showed that not only the parahydrogen itself but also isotopic impurities such as orthohydrogen and deuterated hydrogen in parahydrogen crystals exhibit sharp absorption features. ${ }^{8,9}$ The sharpest transition so far observed is the absorption of deuterated hydrogen, whose width is only $4 \mathrm{MHz}$ (full width at half maximum, FWHM). ${ }^{8}$ The width is almost two orders of magnitude narrower than that of Doppler-limited gas phase spectra.
The sharp linewidth indicates that parahydrogen crystals are a promising medium for high-resolution matrix isolation spectroscopy. ${ }^{7,10}$ Following the work of the group in Chicago, the authors' group in Kyoto ${ }^{11}$ and Fajardo's group at Edwards Air Force Base ${ }^{12,13}$ have independently initiated high-resolution spectroscopic studies of atoms and molecules embedded in parahydrogen crystals. We showed that most of the spectral widths of molecules in parahydrogen crystals are sharper than $0.01 \mathrm{~cm}^{-1}$ at low temperatures. ${ }^{14}$ The spectral resolution of $0.01 \mathrm{~cm}^{-1}$ is high enough to discuss intermolecular interactions and molecular motions in the condensed phase in great detail. ${ }^{11}$

In a series of papers we have extensively studied rotation-vibration transitions of methane molecules embedded in parahydrogen crystals by high-resolution infrared absorption spectroscopy. ${ }^{14-19}$ The analysis of observed spectra reveals that the rotational energy levels of the methane is fully quantized, having the rotational quantum number $J$ as a good quantum number. Here, we again discuss methane molecules in parahydrogen crystals, but we focus on the nuclear spin conversion of methane.

In the case of $\mathrm{CH}_{4}$, the four equivalent protons can be coupled into three nuclear spin states, $I=0,1$, and 2 . The Pauli principle requires that only certain nuclear spin wave functions couple with any particular electron-vibrationrotation wave-functions. ${ }^{20,21}$ As a result, the $J=0$ rotational state is associated with the $I=2$ nuclear spin quintet state and the $J=1$ state is the $I=1$ triplet state, while the $J=2$ state is coupled with both the $I=1$ triplet state and the $I=0$ singlet state. Even if the temperature is lowered sufficiently, the equilibrium distribution cannot be achieved because conversion among the different nuclear spin states is forbidden. ${ }^{22}$ Only weak nuclear spin-nuclear spin magnetic interaction and spin-rotation interaction may cause the conversion among different nuclear spin states in the gas phase. $^{23-25}$ In condensed phases, evidence for the triplet ( $I$ 
$=1) \rightarrow$ quintet $(I=2)$ transition in solid methane has been observed by proton magnetic resonance spectroscopy. ${ }^{26-34}$ The transition of methane in solid argon and krypton has been observed by infrared spectroscopy. ${ }^{35,36}$ A conversion time of about 100 min has been reported in these condensed phases, which is still a slow process compared with other relaxations.

In a previous paper ${ }^{16}$ we briefly reported the fact that the $J=1$ rotational level is populated in spite of the null Boltzmann factor at the observed temperature, and that the population of the $J=1$ rotational level decreases with time, which can be attributed to a relaxation of rotational energy accompanying a nuclear spin conversion. The present article presents additional data and arguments to support the view that the existence of impurity orthohydrogen in parahydrogen crystal increases the conversion rate.

In Sec. 2 we briefly describe the properties of parahydrogen crystals to demonstrate the usefulness of parahydrogen matrices for isolation spectroscopy. Experimental details are given in Sec. 3. In Sec. 4, infrared absorption spectra of $\mathrm{CH}_{4}$ embedded in parahydrogen crystals and their analyses are briefly overviewed. Analysis and discussion on the nuclear spin conversion are given in Sec. 5.

\section{PARAHYDROGEN MATRIX}

There are two kinds of hydrogen molecules existing in nature: para- and orthohydrogen. The parahydrogen molecule possesses a nuclear spin angular momentum of $I=0$, while for orthohydrogen $I=1$. Because the total wave function of $\mathrm{H}_{2}$ has to be antisymmetric with respect to the permutation of hydrogen atoms, parahydrogen in its ground electronic state is associated with the rotational states of even quantum numbers, while orthohydrogen is associated with odd numbers. Since the interconversion between $I=0$ and $I=1$ nuclear spin states is very slow in the absence of an external magnetic field, the parahydrogen and orthohydrogen can be considered to be different molecules under normal conditions. Since the rotational constants of hydrogen molecules is as large as $60 \mathrm{~cm}^{-1}$ (Ref. 37), the para- and orthohydrogen occupy rotational quantum numbers of $J=0$ and $J=1$, respectively, at liquid He temperatures. Herein, the terms para- and orthohydrogen are used to signify hydrogen molecules with $J=0$ and $J=1$, respectively.

Since parahydrogen, with the rotational quantum number $J=0$, has no permanent electric moments of any order, we consider the molecule to be spherical, like rare gas atoms. Due to the spherical nature of parahydrogen, the crystal of parahydrogen provides a homogeneous environment for a guest molecule. On the other hand, orthohydrogen, with the rotational quantum number $J=1$, has a permanent quadrupole moment. ${ }^{38}$ Thus the interaction influenced by orthohydrogen is stronger than that by parahydrogen. From the spectroscopic point of view, the existence of orthohydrogen in the crystal causes additional broadening due to the quadrupolar interaction. ${ }^{8}$ Therefore, it is desired that the concentration of orthohydrogen be as low as possible. The concentration of orthohydrogen can be reduced to less than $0.05 \%$ by using an ortho-para converter ${ }^{11,13}$ operated at $13.8 \mathrm{~K}$.

The crystal structure of pure parahydrogen is a complete hexagonal close-packed (hcp), as has been proved spectro- scopically. ${ }^{6}$ The lattice constant of solid hydrogen $(3.78 \AA)$ is considerably larger than that of $\mathrm{Ne}(3.16 \AA)$. The large lattice constant of hydrogen results from large zero-point lattice vibration due to the small mass of $\mathrm{H}_{2}$. The large lattice constant of parahydrogen provides more free space for a guest molecule as compared with other matrices.

The importance of parahydrogen as the matrix for infrared spectroscopy was first proposed by Oka et al. ${ }^{7,10}$ and has been proved by the authors' group ${ }^{11,16}$ and Fajardo's group. $^{12,13}$ Independently, Miyazaki et al. found that the parahydrogen matrix is useful for ESR spectroscopy because parahydrogen does not have any magnetic moments which cause a broadening of ESR linewidths. ${ }^{39}$

Visible and UV spectroscopy of atoms in solid hydrogen has been conducted by Fajardo et al. They have studied the reactive dynamics of dopants in solid hydrogen with the ultimate aim of finding high-performance rocket propellants. ${ }^{40}$

Infrared studies of rovibrational transitions of molecules isolated in parahydrogen crystals have been developed by the authors' group and Fajardo's group, independently. Techniques for making parahydrogen crystals in two groups are different. In Kyoto, we made the crystals in an enclosed cell, as is described in the next section. ${ }^{11,16}$ In the Edwards Air Force Base research, Fajardo developed a technique to grow transparent crystals on a cold surface in an open vacuum. Due to the relatively high vapor pressure of $\mathrm{H}_{2}$ even at liquid He temperatures, ${ }^{41}$ the standard deposition technique which is usually employed for isolation spectroscopy of rare gas matrices can not be applied straightforwardly. Fajardo found a condition for growing completely transparent crystals of millimeter thickness by controlling the deposition rate and the temperature of the substrate. ${ }^{13}$ On the other hand, our enclosed cell technique allows us to grow crystals at a higher temperature, which maintains the equilibrium between gas and solid phases without encountering the problem of vaporization of samples.

The two methods have their own advantages and disadvantages. The advantage of growing the crystal in an enclosed cell is that the crystal structure surrounding the guest molecules becomes completely hcp. ${ }^{16}$ Therefore the fine structure of the observed spectra in an enclosed cell can be treated by a quantitative analysis of the molecular interaction and molecular motions in the condensed phase based on first principles. Crystals grown by Fajardo's deposition technique are found to be a mixture of hcp and fcc structures. ${ }^{19}$ The different environment surrounding embedded molecules causes extra transitions, which makes the quantitative analysis of the spectra more difficult. On the other hand, one can dope any molecules in solid hydrogen by the deposition technique, while a very limited number of molecules can be isolated by our enclosed cell technique.

\section{EXPERIMENTS}

Parahydrogen crystals were grown in a cylindrical copper cell with both ends enclosed by $\mathrm{BaF}_{2}$ windows with indium gaskets. Pure parahydrogen gas containing less than $0.05 \%$ orthohydrogen was obtained by passing high purity $(>99.9995 \%)$ normal hydrogen gas through an ortho-para converter at $14 \mathrm{~K}$. A detail of the converter is given in a previous review article. ${ }^{11}$ About $10 \mathrm{ppm}$ of methane was 
mixed with the converted hydrogen gas at room temperature. Concentrations of orthohydrogen higher than $0.05 \%$ were controlled by adding normal hydrogen to the converted parahydrogen gas. Then, the mixed gas was continuously introduced into the copper cell installed under the cold surface of a standard Dewar-type liquid He cryostat. The temperature of the cell was kept at $8 \mathrm{~K}$ during the crystal growth, which takes about 2 hours. The typical flow rate of the gas was 100 $\mathrm{cm}^{3} / \mathrm{min}$. The crystal, which was completely transparent, was grown from the copper wall toward the inside. The crystal thus grown is a completely hexagonal close-packed structure, as is proved by the stimulated Raman gain spectroscopy of the $Q_{1}(0)$ transition $^{6}$ and infrared absorption of methane in the crystal. ${ }^{16,19}$ The $c$ axis of the crystal is along the direction of crystal growth.

Infrared absorption spectra were observed by a Fourier transform infrared (FTIR) spectrometer (Nicolet Magna 750) with a resolution of $0.25 \mathrm{~cm}^{-1}$. A globar source, $\mathrm{KBr}$ beam splitter, and a liquid- $\mathrm{N}_{2}$-cooled $\mathrm{HgCdTe}$ (MCT) detector were used for recordings. All the measurements were done at $4.8 \mathrm{~K}$.

In an experiment to determine the concentration dependence of orthohydrogen impurity on the conversion rate, orthohydrogen molecules were added to the premixed gas in concentrations of $0.05,0.2,2,10,20,30$, and $75 \%$ while maintaining the concentration of $\mathrm{CH}_{4}$. The conversion rate was followed by observing the relative intensities of the FTIR absorption of the lines arising from different nuclear spin states. Several spectra were recorded for each sample intermittently at reasonably separated times. One recording took about 10 minutes. During the interval of the recordings, the globar source was turned off in order to avoid conversion due to photoexcitation by the globar light.

\section{OBSERVED SPECTRA AND ROVIBRATIONAL ENERGY LEVELS OF METHANE}

Since the intermolecular distance of solid hydrogen of $3.78 \AA$ is significantly larger than the van der Waals diameter of methane at about $3.24 \AA$, methane molecules can rotate almost freely in para-hydrogen crystals. ${ }^{42}$ In previous papers, we have shown that the rotational quantum number $J$ of the methane is still a good quantum number in parahydrogen crystals and that the effective rotational constant is only $10 \%$ smaller than that in free space. ${ }^{15,16,18}$

Figure 1 shows an FTIR spectrum of the $\nu_{3}$ band of methane in a parahydrogen crystal. The orthohydrogen concentration is $0.05 \%$. The large splitting, of about $9 \mathrm{~cm}^{-1}$, is assigned to the rotational branches of methane; the transitions at around $3008 \mathrm{~cm}^{-1}$ are assigned to $P(1)$, those at around $3017 \mathrm{~cm}^{-1}$ to $Q(1)$, those at around $3025 \mathrm{~cm}^{-1}$ to $R(0)$, and those at higher than $3031 \mathrm{~cm}^{-1}$ to $R(1)$. The small splittings of $0.5 \mathrm{~cm}^{-1}$ appearing in each rotational branch are due to the $M$ quantum number of methane, which is the projection of the rotational quantum number $J$ along the crystal axis. The splitting of the $M$-sublevels is caused by the crystal field of solid parahydrogen.

All the transitions appearing in Fig. 1 can be quantitatively interpreted by assuming that methane occupies a substitutional site of the hcp crystal structure of solid hydrogen, and the methane, having $T_{d}$ symmetry, rotates freely under

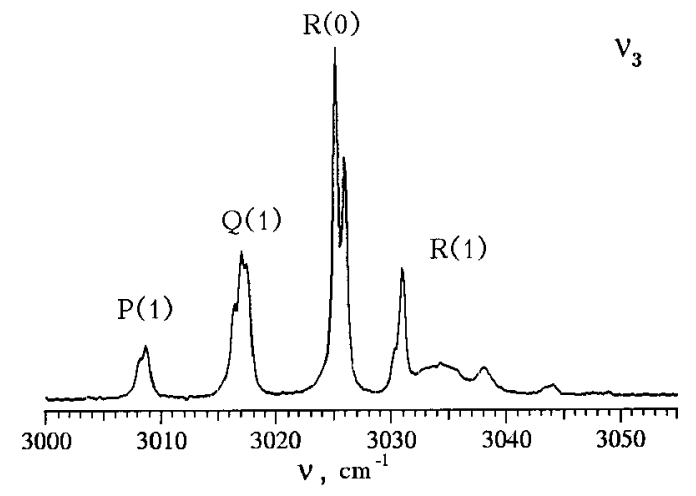

FIG. 1. Infrared absorption spectrum of the $\nu_{3}$ transition of $\mathrm{CH}_{4}$ embedded in a parahydrogen crystal. The orthohydrogen concentration is $0.05 \%$. The spectral resolution is $0.25 \mathrm{~cm}^{-1}$.

the crystal field of $D_{3 h}$ symmetry. In this case, the first anisotropic crystal field potential is found to be

$$
\begin{aligned}
V(\Omega)= & \varepsilon_{3 c} \frac{1}{2}\left[D_{2,3}^{(3)}(\Omega)-D_{-2,3}^{(3)}(\Omega)\right. \\
& \left.+D_{2,-3}^{(3)}(\Omega)-D_{-2,-3}^{(3)}(\Omega)\right] \\
= & \varepsilon_{3 c} \frac{\sqrt{6}}{4}[-2 \cos 2 \chi \cos \theta \cos 3 \varphi \\
& \left.+\sin 2 \chi\left(1+\cos ^{2} \theta\right) \sin 3 \varphi\right] \sin \theta
\end{aligned}
$$

where $\Omega \equiv(\chi, \theta, \varphi)$ is the Euler angle of methane relative to the crystal axis, and $D_{m, n}^{(l)}(\Omega)$ is Wigner's rotation matrix. ${ }^{43}$ The definition of the Euler angles and of Wigner's rotation matrix is the same as employed by Hougen. ${ }^{44}$ The symbol $\varepsilon_{3 c}$ is a crystal-field parameter to be determined by analysis of the observed spectra. Equation (1) can be easily derived with the use of the extended group theory. ${ }^{15,45}$

The interaction potential $V(\Omega)$ in Eq. (1) causes the splittings of degenerate $M$-sublevels of the spherical rotor in the free space. The rotational energy levels in the ground vibrational state can be calculated as the eigenvalues of the matrix of Hamiltonian $H=B^{\prime \prime} J^{2}+V(\Omega)$ where $J$ is the rotational angular momentum operator, and $B^{\prime \prime}$ is the rotational constant of the ground state. The rotational levels in the triply degenerate excited vibrational states can be obtained by taking into account the Coriolis interaction in addition to the rotational Hamiltonian. In a previous paper, we have determined molecular constants of methane and the crystal field parameter, $\varepsilon_{3 c}$, by the least-squares fitting of the observed transition wave numbers with the use of the crystal-field potential given in Eq. (1) ${ }^{16}$ Refer to our previous papers for a complete analysis. ${ }^{15,16}$

It should be noted that all the observed absorption lines can be assigned to the rotational branches, and thus the socalled rotationless transition ${ }^{46,47}$ is absent in our spectrum. Rotationless transition have been observed in the case of water isolated in rare gas matrices. ${ }^{46,47}$ Recently, it was observed that $\mathrm{H}_{2} \mathrm{O}$ in solid parahydrogen also exhibits a rotationless transition. ${ }^{48}$ The presence of rotationless transitions were interpreted as indicating that the molecules are firmly trapped in interstitial sites of the lattice. The absence of the rotationless transition in the case of methane in parahydro- 

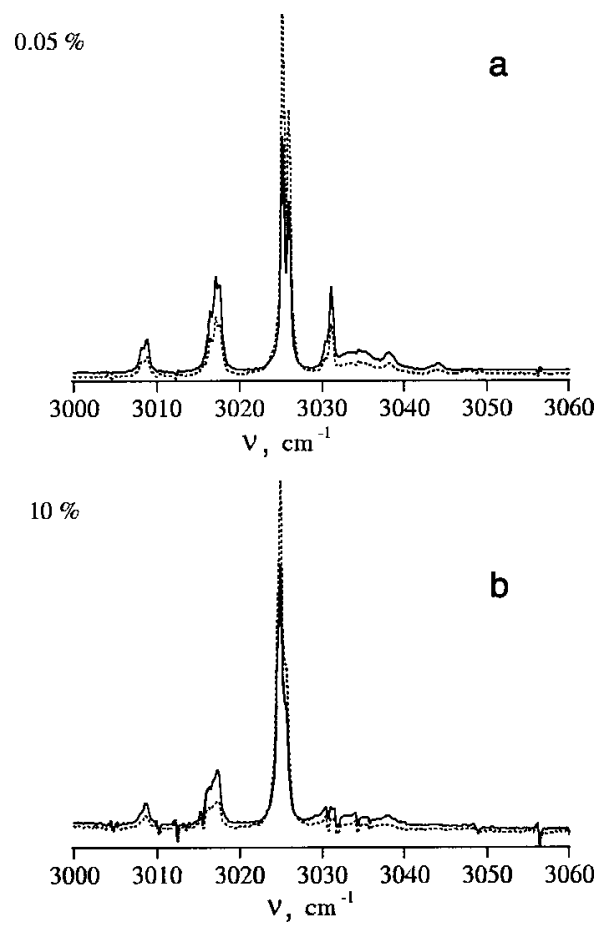

FIG. 2. Temporal behavior of the $\nu_{3}$ transition of $\mathrm{CH}_{4}$ in para-hydrogen crystals with orthohydrogen concentration of $0.05 \%$ (a) and $10 \%$ (b). The solid line is the spectrum observed just after crystal growth. The dotted line is the spectrum observed 2 hours after crystal growth. The sharp spikes are due to the absorption of moisture in the air.

gen crystals confirms that methane occupies a substitutional site of the crystal, but not any interstitial sites.

Figure 2 represents a time-dependent change of spectral structure for the $\nu_{3}$ transition (spectra of crystals with the ortho concentration of $0.05 \%$ and $10 \%$, respectively). The solid line is the spectrum observed immediately after the growth of the crystal, while the dotted line is the spectrum observed 2 hours after crystal growth. It is clearly seen that all the absorptions except those at around $3025 \mathrm{~cm}^{-1}$ become weak after a few hours, while the absorptions around 3025 $\mathrm{cm}^{-1}$ become strong.

In Table I the observed integrated intensities of the $R(0)$ and $Q(1)$ transitions at various times are given for the crystals with orthohydrogen concentrations of $0.05,0.2,2.0,10$, 20,30 , and $75 \%$, respectively. Note that the time when we finished making the crystal was taken as the origin of time $(t=0)$ given in the second column. It is observed that the integrated intensity of the $R(0)$ transition increases with time, while that of the $Q(1)$ transition decreases. The timedependent absorption changes are due to the nuclear spin conversion of methane. In the next section we discuss the conversion in detail.

\section{NUCLEAR SPIN CONVERSION}

It is convenient to review the salient features of the nuclear spin modification of the methane molecule. The Pauli principle requires the total wave-function of molecules to be antisymmetric with respect to the permutation of any identical nuclei. It follows the fact that only certain nuclear spin wave function couples with any particular electronvibration-rotation wave functions. The symmetry of the ro-
TABLE I. Time-dependent absorption intensities of $\mathrm{CH}_{4}$ in parahydrogen crystals.

\begin{tabular}{|c|c|c|c|c|c|}
\hline $\begin{array}{c}\text { ortho } \\
\text { concentration, } \\
\%\end{array}$ & Time, $\min ^{a}$ & $I[R(0)]^{b}$ & $I[Q(\mathbf{1})]^{b}$ & Sum $^{c}$ & $c(t)^{d}$ \\
\hline \multirow[t]{6}{*}{0.05} & 50 & 0.104 & 0.041 & 0.223 & 0.467 \\
\hline & 85 & 0.113 & 0.038 & 0.223 & 0.506 \\
\hline & 175 & 0.137 & 0.031 & 0.227 & 0.604 \\
\hline & 205 & 0.140 & 0.030 & 0.227 & 0.617 \\
\hline & 270 & 0.152 & 0.027 & 0.230 & 0.660 \\
\hline & 340 & 0.161 & 0.024 & 0.231 & 0.698 \\
\hline \multirow[t]{3}{*}{0.2} & 32 & 0.044 & 0.020 & 0.104 & 0.429 \\
\hline & 92 & 0.046 & 0.015 & 0.088 & 0.521 \\
\hline & 152 & 0.052 & 0.014 & 0.091 & 0.568 \\
\hline \multirow[t]{5}{*}{2.0} & 50 & 0.122 & 0.048 & 0.261 & 0.467 \\
\hline & 100 & 0.138 & 0.042 & 0.260 & 0.531 \\
\hline & 160 & 0.151 & 0.036 & 0.255 & 0.591 \\
\hline & 220 & 0.161 & 0.033 & 0.257 & 0.627 \\
\hline & 270 & 0.171 & 0.030 & 0.258 & 0.663 \\
\hline \multirow[t]{6}{*}{10} & 60 & 0.146 & 0.046 & 0.279 & 0.523 \\
\hline & 115 & 0.172 & 0.040 & 0.288 & 0.597 \\
\hline & 160 & 0.184 & 0.034 & 0.283 & 0.651 \\
\hline & 220 & 0.199 & 0.029 & 0.283 & 0.703 \\
\hline & 280 & 0.209 & 0.023 & 0.276 & 0.758 \\
\hline & 340 & 0.205 & 0.022 & 0.269 & 0.763 \\
\hline \multirow[t]{4}{*}{20} & 14 & 0.143 & 0.038 & 0.253 & 0.565 \\
\hline & 74 & 0.179 & 0.027 & 0.257 & 0.696 \\
\hline & 134 & 0.191 & 0.020 & 0.249 & 0.767 \\
\hline & 194 & 0.198 & 0.018 & 0.250 & 0.791 \\
\hline \multirow[t]{5}{*}{30} & 15 & 0.598 & 0.147 & 1.024 & 0.584 \\
\hline & 75 & 0.751 & 0.092 & 1.018 & 0.738 \\
\hline & 135 & 0.795 & 0.074 & 1.010 & 0.787 \\
\hline & 195 & 0.809 & 0.067 & 1.003 & 0.806 \\
\hline & 255 & 0.813 & 0.065 & 1.002 & 0.812 \\
\hline \multirow[t]{4}{*}{75} & 6 & 0.439 & 0.052 & 0.590 & 0.744 \\
\hline & 36 & 0.472 & 0.045 & 0.603 & 0.783 \\
\hline & 66 & 0.476 & 0.043 & 0.601 & 0.792 \\
\hline & 96 & 0.475 & 0.042 & 0.597 & 0.796 \\
\hline
\end{tabular}

$\overline{{ }^{a}}$ The time when we finished making the crystals was taken as the origin of time $(t=0)$.

${ }^{b}$ Integrated intensity of all $M$-sublevels of each transition.

${ }^{c}$ The value of $I[R(0)]+2.9 I[Q(1)]$, which is supposed to be a constant irrespective of time after the crystal growth (see text). The absolute value of the sum varies depending on the concentration of $\mathrm{CH}_{4}$ in crystals.

${ }^{d}$ The mole fraction of $J=0$ methane, defined in Eq. (2).

tational wave function of a $\mathrm{CH}_{4}$ molecule is connected to the symmetry of the nuclear spin wave function by the requirement that the total wave function be antisymmetric with re- 
spect to the interchange of any two protons. It was fully discussed by Wilson ${ }^{20}$ that the requirement is met if both rotation and spin wave functions belong to the same representation in the pure rotational tetrahedral $(T)$ point group. The two $I=0$ states

$$
\begin{gathered}
12^{-1 / 2}[2|\alpha \alpha \beta \beta\rangle+2|\beta \beta \alpha \alpha\rangle-|\alpha \beta \alpha \beta\rangle-|\beta \alpha \alpha \beta\rangle \\
-|\alpha \beta \beta \alpha\rangle-1 \beta \alpha \beta \alpha\rangle], \\
4^{-1 / 2}[|\alpha \beta \alpha \beta\rangle+|\beta \alpha \beta \alpha\rangle-|\alpha \beta \beta \alpha\rangle-|\beta \alpha \alpha \beta\rangle]
\end{gathered}
$$

belong to the irreducible representation $E$, the nine $I=1$ states

$$
\begin{aligned}
& 4^{-1 / 2}[|\alpha \alpha \alpha \beta\rangle-|\alpha \alpha \beta \alpha\rangle+|\alpha \beta \alpha \alpha\rangle-|\beta \alpha \alpha \alpha\rangle], \\
& 2^{-1 / 2}[|\alpha \beta \alpha \beta\rangle-|\beta \alpha \beta \alpha\rangle], \\
& 4^{-1 / 2}[|\beta \beta \beta \alpha\rangle-|\beta \beta \alpha \beta\rangle+|\beta \alpha \beta \beta\rangle-|\alpha \beta \beta \beta\rangle], \\
& 4^{-1 / 2}[|\alpha \alpha \alpha \beta\rangle-|\alpha \alpha \beta \alpha\rangle-|\alpha \beta \alpha \alpha\rangle+|\beta \alpha \alpha \alpha\rangle], \\
& 2^{-1 / 2}[|\alpha \beta \beta \alpha\rangle-|\beta \alpha \alpha \beta\rangle], \\
& 4^{-1 / 2}[|\beta \beta \beta \alpha\rangle-|\beta \beta \alpha \beta\rangle-|\beta \alpha \beta \beta\rangle+|\alpha \beta \beta \beta\rangle], \\
& 4^{-1 / 2}[|\alpha \alpha \alpha \beta\rangle+|\alpha \alpha \beta \alpha\rangle-|\alpha \beta \alpha \alpha\rangle-|\beta \alpha \alpha \alpha\rangle], \\
& 2^{-1 / 2}[|\alpha \alpha \beta \beta\rangle-|\beta \beta \alpha \alpha\rangle], \\
& 4^{-1 / 2}[|\beta \beta \beta \alpha\rangle+|\beta \beta \alpha \beta\rangle-|\beta \alpha \beta \beta\rangle-|\alpha \beta \beta \beta\rangle]
\end{aligned}
$$

belong to the irreducible representation $F$, and the five $I$ $=2$ states

$$
\begin{aligned}
& |\alpha \alpha \alpha \alpha\rangle, \\
& 4^{-1 / 2}[|\alpha \alpha \alpha \beta\rangle+|\alpha \alpha \beta \alpha\rangle+|\alpha \beta \alpha \alpha\rangle+|\beta \alpha \alpha \alpha\rangle], \\
& 6^{-1 / 2}[|\alpha \alpha \beta \beta\rangle+|\beta \beta \alpha \alpha\rangle+|\alpha \beta \alpha \beta\rangle+|\beta \alpha \beta \alpha\rangle \\
& \quad+|\beta \alpha \alpha \beta\rangle+\mid \alpha \beta \beta \alpha], \\
& 4^{-1 / 2}[|\beta \beta \beta \alpha\rangle+|\beta \beta \alpha \beta\rangle+|\beta \alpha \beta \beta\rangle+|\alpha \beta \beta \beta\rangle],
\end{aligned}
$$

$|\beta \beta \beta \beta\rangle$

belong to the irreducible representation $A$. Therefore, the $J$ $=0$ rotational state having the $A$ representation in $T$ is combined with $I=2(A)$ spin states, the $J=1$ rotational states having the $F$ representation are combined with $I=1(F)$ spin states, and the $J=2$ rotational states having the $E$ and $F$ representations are combined with $I=0(E)$ and $I=1(F)$ spin states, respectively.

The same coupling between rotation and nuclear spin

\begin{tabular}{|c|c|c|c|c|c|c|}
\hline \multirow{2}{*}{$J$} & \multirow{2}{*}{$\Gamma_{\text {tot }}^{a}$} & \multirow{2}{*}{$I$} & \multirow{2}{*}{$\begin{array}{c}\text { Statistical } \\
\text { weight }\end{array}$} & \multirow{2}{*}{$\begin{array}{c}\text { Term value } \\
\mathrm{cm}^{-1}\end{array}$} & \multicolumn{2}{|c|}{$\begin{array}{c}\text { Equilibrium } \\
\text { distribution } \\
\text { at } 5 \mathrm{~K}\end{array}$} \\
\hline & & & & & $\begin{array}{l}\text { without } \\
\text { NSC }^{c}\end{array}$ & $\begin{array}{c}\text { after } \\
\text { NSC }^{d}\end{array}$ \\
\hline 2 & $E \bar{E}$ & 0 & 4 & 36.448 & 0.0 & 0.0 \\
\hline 2 & $E \overline{F_{2}}$ & 1 & 6 & 31.426 & 0.0 & 0.0 \\
\hline 2 & $A_{1} \bar{E}$ & 0 & 2 & 29.527 & 0.01 & 0.0 \\
\hline 2 & $E \overline{F_{2}}$ & 1 & 6 & 28.828 & 0.0 & 0.0 \\
\hline 2 & $A_{1} \overline{F_{2}}$ & 1 & 3 & 27.789 & 0.0 & 0.0 \\
\hline 2 & $E \bar{E}$ & 0 & 4 & 23.501 & 0.12 & 0.0 \\
\hline 1 & $A_{2} \bar{F}_{1}$ & 1 & 3 & 9.485 & 0.16 & 0.03 \\
\hline 1 & $E \overline{F_{2}}$ & 1 & 6 & 8.815 & 0.40 & 0.08 \\
\hline 0 & $A_{1} \overline{A_{1}}$ & 2 & 5 & 0.000 & 0.31 & 0.89 \\
\hline
\end{tabular}
wave functions is required also for a $\mathrm{CH}_{4}$ molecule in a crystal, since the symmetry of space does not affect on the symmetry of the permutation of nuclei within a molecule. In Table II, the representation of the rotational wave function and the coupled nuclear spin state are listed for $J=0,1$, and 2 levels of the ground vibrational state. The first, second, and third columns show the total rotational angular momentum $J$, the representation of the rotational wave function in the extended group $G,{ }^{15}$ and the total nuclear spin angular momentum $I$, respectively. The $J=0$ rotational state is combined with $I=2$ spin states, the $J=1$ rotational states are combined with $I=1$ spin states, and the $J=2$ rotational states are combined with either $I=0$ or $I=1$ spin states. The fourth column shows the statistical weight of each level. ${ }^{20}$
TABLE II. Energies and Boltzmann distribution of the vibrational ground state of $\mathrm{CH}_{4}$ in parahydrogen crystals.

${ }^{a}$ Representation of the rotational wave function in the extended group $G={ }^{\prime} D_{3 h}^{\prime} \otimes \overline{G_{24}^{\prime}}$ (See Ref. 15).

${ }^{b}$ Calculated with the molecular parameters obtained in Ref. 15.

${ }^{c}$ Population of each rotational level at $4.8 \mathrm{~K}$ if the nuclear spin conversion is completely forbidden. The ratio of the $A, F$, and $E$ nuclear spin states is assumed to be 5:9:2 at room temperature. ${ }^{22}$

${ }^{d}$ Population of each rotational level at $4.8 \mathrm{~K}$ without the nuclear spin modification.

The fifth column of Table II shows the energies of the ground vibrational state of $\mathrm{CH}_{4}$ in parahydrogen crystals, calculated using the previously determined parameters $B$ $=4.793 \mathrm{~cm}^{-1}$ and $\varepsilon_{3 c}=-25.8 \mathrm{~cm}^{-1}$. Together with the statistical weight in the fourth column, the population of each state at any temperature can be calculated. The equilibrium distribution at $4.8 \mathrm{~K}$ with and without the nuclear spin conversion is given in the sixth and seventh columns of Table II. Without the nuclear spin modification, the equilibrium Boltzmann distribution of the ground rotational levels at $4.8 \mathrm{~K}$ has to be $0.89,0.08$, and 0.03 for the $J=0$ level, $J=1, M$ $=1$ level, and $J=1, M=0$ level, respectively. However, as is seen in Fig. 1, the spectral intensity of $P(1), Q(1)$, and $R(1)$, all of which are transitions from the $J=1$ levels, are apparently stronger than the intensity predicted from the equilibrium distribution given in the seventh column in Table II. This indicates that there is an appreciable population of the $J=1$ levels just after the crystal growth, contrary to the $4.8 \mathrm{~K}$ Boltzmann distribution. The non-Boltzmann distribution is due to the nuclear spin modification.

It should be noted that no absorption from the $J=2$ rotational states was observed at any time, as is seen in Fig. 2, although the lowest rotational state coupled with the $I=0$ nuclear spin state is the $J=2$ state. There has to be an appreciable population in the $J=2$ levels just after the cooling 
of the temperature of $\mathrm{CH}_{4}$, as is seen in Table $\mathrm{II},{ }^{22}$ but we have never observed transitions from the $J=2$ levels. The absence of the $J=2$ population may be explained by fast relaxation from the $J=2$ levels to $J=1$. The $J=2$ rotational state is coupled not only with the $I=0$ nuclear spin state but also with the $I=1$ nuclear spin states. Due to the proximity between the $I=0$ and $I=1$ in the $J=2$ level, the nuclear spin-rotation interaction could yield mixing between the $I$ $=0$ and $I=1$ nuclear spin states, which results in the fast relaxation from the $J=2$ to $J=1$ rotational states. The relaxation might be too fast to observe on our experimental time scale. In the following, we consider only the conversion from the $J=1$ levels to $J=0$.

In order to discuss the nuclear spin conversion process quantitatively, we define the mole fraction of the $J=0$ state as

$$
c(t)=[J=0]_{t} /\left([J=0]_{t}+[J=1]_{t}\right)
$$

where $[J=0]_{t}$ represents the concentration of $J=0$ molecules at time $t$. The mole fraction is related to the integrated absorption intensities as

$$
c(t)=I[R(0)]_{t} /\left(I[R(0)]_{t}+A I[Q(1)]_{t}\right)
$$

where $I[R(0)]_{t}$ and $I[Q(1)]_{t}$ are the integrated intensities of the $R(0)$ and $Q(1)$ transitions, respectively, and $A$ is a constant which is equal to the ratio of the transition probabilities of $R(0)$ and $Q(1)$. Although we do not know the transition probability exactly, we can estimate it by the fact that the sum of $[J=0]_{t}+[J=1]_{t}$, which is proportional to $I[R(0)]_{t}+A I[Q(1)]_{t}$, should be constant at all times. We found that the value of $A=2.9$ gives approximately constant values of $I[R(0)]_{t}+A I[Q(1)]_{t}$ as is seen in the fifth column of Table I. We therefore assume here that the $R(0)$ transition is 2.9 times stronger than the $Q(1)$ transition for the $\nu_{3}$ mode of $\mathrm{CH}_{4}$ in parahydrogen crystals. With the assumption of $A=2.9$, the mole fraction at any given time is obtained as shown in the last column of Table I.

The change of the mole fraction with time is plotted in Fig. 3 for different orthohydrogen concentrations. If we treat the time-dependent change of the methane absorption spectra with first-order kinetics, the time dependence of the mole fraction $c(t)$ may be written as

$$
c(t)=[c(0)-c(\infty)] \exp (-k t)+c(\infty),
$$

where $k$ is the sum of the $J=1 \rightarrow J=0$ rate and the $J=1$ $\leftarrow J=0$ rate. In Fig. 3 the best-fit functions of the form of Eq. (4) are also drawn for each concentration. Here the value of $c(\infty)=0.89$, given in Table II as the equilibrium distribution, was assumed for the samples with low orthohydrogen concentration $(\leqslant 10 \%)$. For higher orthohydrogen concentration $(\geqslant 10 \%)$, we treated the $c(\infty)$ as a parameter of the fitting. ${ }^{49}$ The conversion rate $k$ defined in Eq. (4) obtained from the least-squares fitting method are plotted in Fig. 4 as a function of the orthohydrogen concentration.

The first-order kinetics in Eq. (4) gives acceptable agreement with all the experimental time dependence, as is seen in Fig. 3. This indicates that the simple first-order kinetics is appropriate for the nuclear spin conversion of methane in parahydrogen crystals.

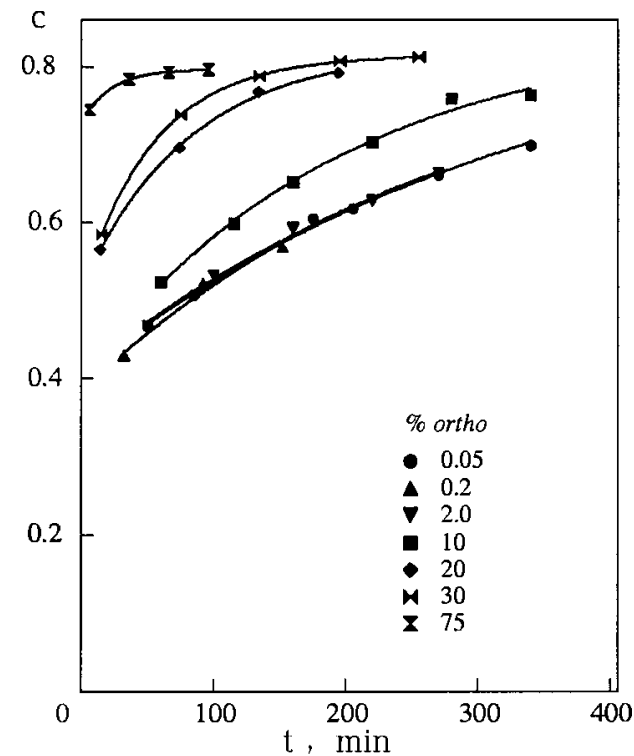

FIG. 3. The time-dependent behavior of $J=0$ methane molecules at various orthohydrogen concentrations. The solid lines are the theoretical curves of Eq. (4).

It is seen in Figs. 3 and 4 that the conversion rate is unchanged by the addition of up to $2 \%$ orthohydrogen but increases with more than $10 \%$ orthohydrogen. The fact that the conversion rate increases with increasing orthohydrogen concentration indicates that the nuclear spin conversion is enhanced in the presence of orthohydrogen molecules. Since the orthohydrogen has a magnetic moment, ${ }^{38}$ the magnetic field from the orthohydrogen enforces the forbidden spin relaxation from $I=1$ to $I=2$.

In order to obtain a qualitative picture of the effect of orthohydrogen concentration on the spin conversion rate, it is necessary to know the number and distances of orthohydrogen molecules from a methane molecule. Here we approximate the number of orthohydrogen molecules on the assumption of a homogeneous distribution of orthohydrogen and methane molecules in the crystal. If we assume that the crys-

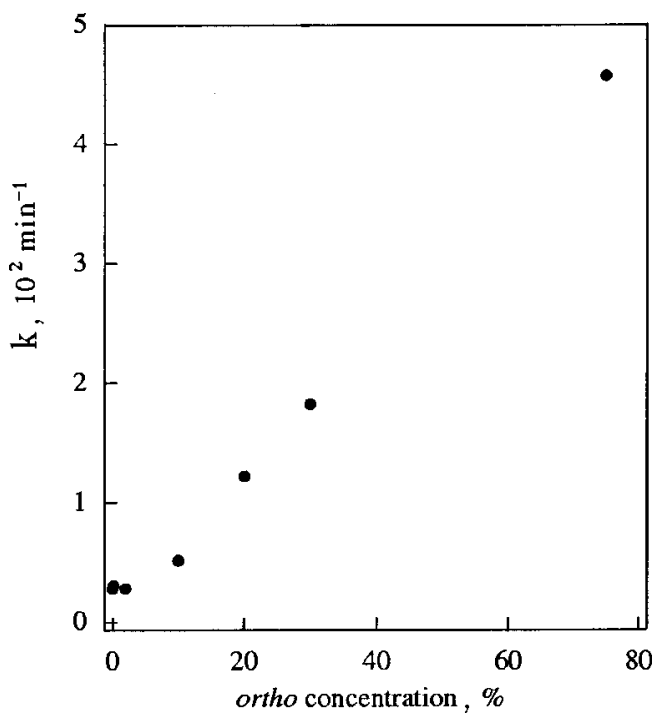

FIG. 4. Dependence of the conversion rate upon orthohydrogen concentration. To obtain the conversion rate, the first-order kinetics of Eq. (4) was assumed. 
tal is a hexagonal close-packed structure whose nearestneighbor distance is $3.783 \AA$, it is roughly obtained that the average distances between methane and orthohydrogen molecules are $9.8 \AA, 7.2 \AA$, and $6.2 \AA$ for concentrations of 1,2 , and $5 \%$, respectively. On the other hand, one orthohydrogen molecule always exists next to methane in the case of $8 \%$, and two or more orthohydrogen molecules exist next to methane for more than $16 \%$.

The fact that the conversion rate is unchanged below $2 \%$ indicates that the magnetic field of orthohydrogen is only effective when the orthohydrogen is next to methane. The basic interaction which causes nuclear spin conversion is the intermolecular magnetic dipole-dipole interaction between protons, which has been discussed by Wigner for the case of gas phase $\mathrm{H}_{2}$ spin conversion, ${ }^{50}$ by Motizuki and Nagamiya for $\mathrm{H}_{2}$ spin conversion in solid hydrogen ${ }^{51}$, and by Nijman and Berlinsky for $\mathrm{CH}_{4}$ conversion in solid methane. ${ }^{52}$ The distance dependence of the conversion rate in the condensed phase caused by the intermolecular magnetic dipole-dipole interaction has been found to be $R^{-8}$ or higher. ${ }^{51,52}$ Therefore, it is reasonable to consider that the conversion by orthohydrogen is applicable only for nearest neighbors in our case. Consequently, we can conclude that the increase of the conversion rate of above $10 \%$ orthohydrogen is caused by the magnetic dipole-dipole interaction between methane and the nearest-neighbor orthohydrogen molecule(s), while the conversion at lower concentrations is caused by other mechanisms.

The mechanisms of the conversion at lower orthohydrogen concentrations are not yet clear. One possibility is the conversion caused by a strong paramagnetic impurity of $\mathrm{O}_{2}$ molecules. Evidently, trace $\mathrm{O}_{2}$ could not be eliminated in our sample. We guess that the concentration of $\mathrm{O}_{2}$ molecules in our crystal is $10^{-8}$ or less. In order to clarify the role of $\mathrm{O}_{2}$ impurity, the oxygen concentration dependence of the conversion rate has to be observed. However, we would like to note that the observed conversion rate of $3 \times 10^{-3} \mathrm{~min}^{-1}$ at low orthohydrogen concentration is one-half of the conversion rate of $\mathrm{CH}_{4}$ in solid $\mathrm{Ar}$ matrix which is 6 $\times 10^{-3} \mathrm{~min}^{-1}$ (Ref. 36). The observed $6 \times 10^{-3} \mathrm{~min}^{-1}$ rate has been interpreted as due to the spin-spin interaction within the molecule. ${ }^{36}$ Therefore, we believe that the conversion rate of $3 \times 10^{-3} \mathrm{~min}^{-1}$ in solid parahydrogen is likely to be caused by mechanisms other than an impurity of $\mathrm{O}_{2}$ molecules.

The data presented in this article are still preliminary. Further experiments are indispensable for a quantitative discussion on the mechanisms of the nuclear spin conversion of $\mathrm{CH}_{4}$. Since the rotational energy levels of methane in parahydrogen crystals are completely determined, we will be able to discuss not only the basic mechanisms of the conversion, but also more finer details such as rotational $M$-sublevel dependence of the conversion. Since the parahydrogen crystal has been characterized much better than any other crystal, the observed nuclear spin conversion may play a priming role for a deeper understanding of the nuclear spin conversion processes in the condensed phase.

The research herein was supported in part by the Grantsin-Aid for Scientific Research of the Ministry of Education, Science, Culture, and Sports of Japan.
*Email: momose@kuchem.kyoto-u.ac.jp

${ }^{1}$ M. E. Jacox, J. Phys. Chem. Ref. Data 27, 115 (1998).

${ }^{2}$ V. E. Bondybey and V. A. Apkarian, Chem. Phys. 189, 137 (1994).

${ }^{3}$ G. N. Lewis, D. Lipkin, and T. T. Magel, J. Am. Ceram. Soc. 63, 3005 (1941).

${ }^{4}$ E. Whittle, D. A. Dows, and G. C. Pimentel, J. Chem. Phys. 22, 1943 (1954).

${ }^{5}$ M. Okumura, M.-C. Chan, and T. Oka, Phys. Rev. Lett. 62, 32 (1989).

${ }^{6}$ T. Momose, D. P. Weliky, and T. Oka, J. Mol. Spectrosc. 153, 760 (1992).

${ }^{7}$ T. Oka, Annu. Rev. Phys. Chem. 44, 299 (1993).

${ }^{8}$ D. P. Weliky, K. E. Kerr, T. J. Byers, Y. Zhang, T. Momose, and T. Oka, J. Chem. Phys. 105, 4461 (1996).

${ }^{9}$ Y. Zhang, T. J. Byers, M.-C. Chan, T. Momose, K. E. Kerr, D. P. Weliky, and T. Oka, Phys. Rev. B 68, 218 (1998).

${ }^{10}$ M.-C. Chan, Ph.D. Thesis, The University of Chicago (1991).

${ }^{11}$ T. Momose and T. Shida, Bull. Chem. Soc. Jpn. 71, 1 (1998).

${ }^{12}$ M. E. Fajardo and S. Tam, J. Chem. Phys. 108, 4237 (1998).

${ }^{13}$ S. Tam and M. E. Fajardo, Rev. Sci. Instrum. 70, 1926 (1999).

${ }^{14}$ H. Katsuki and T. Momose, Phys. Rev. Lett. 84, 3286 (2000).

${ }^{15}$ T. Momose, J. Chem. Phys. 107, 7695 (1997).

${ }^{16}$ T. Momose, M. Miki, T. Wakabayashi, T. Shida, M.-C. Chan, S. S. Lee, and T. Oka, J. Chem. Phys. 107, 7707 (1997).

${ }^{17}$ T. Momose, H. Katsuki, H. Hoshina, N. Sogoshi, T. Wakabayashi, and T. Shida, J. Chem. Phys. 107, 7717 (1997).

${ }^{18}$ H. Hoshina, T. Wakabayashi, T. Momose, and T. Shida, J. Chem. Phys. 110, 5728 (1999)

${ }^{19}$ S. Tam and M. E. Fajardo, H. Katsuki, H. Hoshina, T. Wakabayashi, and T. Memose, J. Chem. Phys. 111, 4191 (1999).

${ }^{20}$ E. B. Wilson Jr., J. Chem. Phys. 3, 276 (1935).

${ }^{21}$ A. W. Maue, Ann. Phys. (Leipzig) 20, 555 (1937).

${ }^{22}$ M. Hepp, G. Winnewisser, and K. M. T. Yamada, J. Mol. Spectrosc. 164, 311 (1994).

${ }^{23}$ R. F. Curl Jr., J. V. V. Kasper, and K. S. Pitzer, J. Chem. Phys. 46, 3220 (1966).

${ }^{24}$ P.-N. Yi, I. Ozier, and C. H. Anderson, Phys. Rev. 165, 92 (1968).

${ }^{25}$ I. Ozier, P.-N. Yi, A. Khosla, and N. F. Ramsay, Phys. Rev. Lett. 24, 642 (1970).

${ }^{26}$ R. P. Wolf and W. M. Whitney, Low Temperature Physics, J. G. Daunt et al. (Eds.) (Plenum Press, Inc., New York, 1965).

${ }^{27}$ H. P. Hopkins Jr., P. L. Donoho, and K. S. Pitzer, J. Chem. Phys. 47, 864 (1967).

${ }^{28}$ K. P. Wong, J. D. Noble, M. Bloom, and S. Alexander, J. Magn. Reson. 1, 55 (1969).

${ }^{29}$ P. van Hecke and L. van Gerven, Physica (Amsterdam) 68, 359 (1973).

${ }^{30}$ G. J. Vogt and K. S. Pitzer, J. Chem. Thermodyn. 8, 1011 (1976).

${ }^{31}$ J. E. Piott and W. D. McCormick, Can. J. Phys. 54, 1784 (1976); ibid. 54, 1799 (1976)

${ }^{32}$ K. J. Lushington and J. A. Morrison, Can. J. Phys. 55, 1580 (1977).

${ }^{33}$ J. Higinbotham, B. M. Wood, and R. F. Code, Phys. Lett. A 66, 237 (1978).

${ }^{34}$ S. Buchman, D. Candela, W. T. Vetterling, and R. V. Pound, Phys. Rev. B 26, 1459 (1982).

${ }^{35}$ F. H. Frayer and G. E. Ewing, J. Chem. Phys. 46, 1994 (1967).

${ }^{36}$ F. H. Frayer and G. E. Ewing, J. Chem. Phys. 48, 781 (1968).

${ }^{37}$ G. Herzberg, Molecular Spectra and Molecular Structure, Vol. I, Spectra of Diatomic Molecules, Krieger Publishing Co., Malabar, Florida (1989).

${ }^{38}$ J. van Kranendonk, Solid Hydrogen. Theory of the Properties of Solid $\mathrm{H}_{2}$, $\mathrm{HD}$, and $\mathrm{D}_{2}$ (Plenum Press, Inc., New York, 1983).

${ }^{39}$ T. Miyazaki, K. Yamamoto, and J. Arai, Chem. Phys. Lett. 219, 405 (1994).

${ }^{40}$ M. E. Fajardo, S. Tam, T. L. Thompson, and M. E. Cordonnier, Chem. Phys. 189, 351 (1994).

${ }^{41}$ P. C. Souers, Hydrogen Properties for Fusion Energy, University of California Press, Berkeley (1986).

${ }^{42}$ L. Pauling, Phys. Rev. 36, 430 (1930).

${ }^{43}$ E. P. Wigner, Group Theory, Academic Press, New York (1959).

${ }^{44}$ J. T. Hougen, International Review of Science Physical Chemistry, Vol. 3, Ser. 2, Spectroscopy, p. 75, D. A. Ramsay (Ed.), Butterworths, London (1976).

${ }^{45}$ R. E. Miller and J. C. Decius, J. Chem. Phys. 59, 4871 (1973).

${ }^{46}$ R. L. Redington and D. E. Milligan, J. Chem. Phys. 37, 2162 (1962).

${ }^{47}$ R. L. Redington and D. E. Milligan, J. Chem. Phys. 39, 1276 (1963). 
${ }^{48} \mathrm{~T}$. Momose and T. Oka (in preparation).

${ }^{49}$ Since the interaction between orthohydrogen and methane is much stronger than that between parahydrogen and methane, molecular parameters of methane with orthohydrogen next to it could be different from those of methane surrounded by only parahydrogen molecules. Therefore, the equilibrium value $c(\infty)$ is not necessarily be equal to 0.89 for higher orthohydrogen concentration. The $c(\infty)$ values determined by the least-squares fitting were $0.85(10 \%), 0.82(20 \%), 0.81(30 \%)$, and $0.80(75 \%)$.

${ }^{50}$ E. Wigner, Z. Phys. Chem. Abt. B 23, 28 (1933).

${ }^{51}$ K. Motizuki and T. Nagamiya, J. Phys. Soc. Jpn. 11, 93 (1956).

${ }^{52}$ A. J. Nijman and A. J. Berlinsky, Can. J. Phys. 58, 1049 (1980).

This article was published in English in the original Russian journal. Reproduced here with stylistic changes by the Translation Consultant. 